Experimental febrile seizures increase dendritic complexity of newborn dentate granule cells

Peer-reviewed author version

RAIJMAKERS, Marjolein; CLYNEN, Elke; SMISDOM, Nick; NELISSEN, Sofie; BRONE, Bert; RIGO, Jean-Michel; HOOGLAND, Govert \& SWIJSEN, Ann (2016) Experimental febrile seizures increase dendritic complexity of newborn dentate granule cells. In: EPILEPSIA, 57(5), p. 717-726.

DOI: 10.1111/epi.13357

Handle: http://hdl.handle.net/1942/22544 


\section{Experimental febrile seizures increase dendritic complexity of newborn dentate granule cells}

Marjolein Raijmakers ${ }^{1,2}$, Elke Clynen ${ }^{1}$, Nick Smisdom ${ }^{1,3}$, Sofie Nelissen ${ }^{1}$, Bert Brône ${ }^{1}$, JeanMichel Rigo ${ }^{1, *}$, Govert Hoogland ${ }^{2, \pm}$ and Ann Swijsen ${ }^{1, \pm}$

${ }^{1}$ Biomedical Research Institute BIOMED, Hasselt University, Hasselt, Belgium; ${ }^{2}$ Department of Neurosurgery, School for Mental Health and Neuroscience, Maastricht University Medical Center, Maastricht, The Netherlands; ${ }^{3}$ Flemish Institute for Technological Research, Environmental Risk and Health Unit, Mol, Belgium; ${ }^{\ddagger}$ Both authors contributed equally to this work.

*Corresponding author:

Jean-Michel Rigo

Martelarenlaan 42

B-3500 Hasselt

Belgium

Telephone: $\quad+32(0) 11268004$

Fax: $\quad$ +32 (0) 11269299

Email: jeanmichel.rigo@uhasselt.be

Running title (max 40 characters and spaces in length):

Altered morphology of post-FS born DGC

Key words:

Dendritogenesis

Dentate gyrus

Epileptogenesis

Hyperthermia

Neurogenesis

Spine density

Number of text pages:

Number of words (Including introduction - discussion):

Number of references:

Number of figures:

4

Number of tables: 
Raijmakers et al. | 2

\section{Summary and Key words}

Objective: Febrile seizures (FS) are fever-associated convulsions, being the most common seizure disorder in early childhood. A subgroup of these children later develops epilepsy characterized by a hyperexcitable neuronal network in the hippocampus. Hippocampal excitability is regulated by the hippocampal dentate gyrus (DG) where postnatal neurogenesis occurs. Experimental FS increase the survival of newborn hippocampal dentate granule cells (DGC), yet the significance of this neuronal subpopulation to the hippocampal network remains unclear. In the current study, we characterized the temporal maturation and structural integration of these post-FS born DGC in the DG.

Methods: Experimental FS were induced in 10-day old rat pups. The next day, retroviral particles coding for eGFP were stereotactically injected in the DG to label newborn cells. Histochemical analyses of eGFP expressing DGC were performed one, four and eight weeks later and consisted of: 1 . colocalization with neurodevelopmental markers doublecortin, calretinin and NeuN, 2. quantification of dendritic complexity and 3. quantification of spine density and morphology.

Results: At neither time point were neurodevelopmental markers differently expressed between FS animals and normothermia (NT) controls. One week after treatment DGC from FS animals showed dendrites that were $66 \%$ longer than those from NT controls. At four and eight weeks Sholl analysis of the outer $83 \%$ of the molecular layer showed $20-25 \%$ more intersections in FS animals than in NT controls $(p<0.01)$. Although overall spine density was not affected, an increase in mushroom type spines was observed after eight weeks.

Significance: Experimental FS increase dendritic complexity and the number of mushroom type spines in post-FS born DGC, demonstrating a more mature phenotype and suggesting increased incoming excitatory information. The consequences of this hyperconnectivity to signal processing in the DG and the output of the hippocampus remain to be studied.

Key words: Dendritogenesis, dentate gyrus, epileptogenesis, hyperthermia, neurogenesis, spine density 


\section{Key Points:}

The morphology of newborn dentate granule cells after febrile seizures (FS) is characterized by:

- A stable maturation rate based on expression of neurodevelopmental markers DCX, calretinin and NeuN

- An increased dendritic length at one week after FS induction

- An increased dendritic complexity connecting with the outer $83 \%$ of the molecular layer at four and eight weeks after FS induction

- An increase of mushroom type spines eight weeks after FS induction 
Raijmakers et al. | 4

\section{Introduction}

Febrile seizures (FS) are the most common convulsions in children, with an occurrence of 3 $4 \%$ in the general population between the age of three months and five years ${ }^{1}$. In about $35 \%$ of these children the FS are complex, i.e. prolonged or recurrent, which increases the risk for the development of epilepsy later in life $^{2}$. Experimental complex FS cause a persistent increase in hippocampal excitability resulting in an enhanced seizure susceptibility in adulthood $^{3}$. The cellular mechanisms that underlie this FS-induced epileptogenesis are currently being unraveled, but still not fully understood.

Hippocampal excitability is normally regulated by the hippocampal dentate gyrus (DG), the area where also postnatal neurogenesis occurs. Neurogenesis dynamically controls the hippocampal output as newborn dentate granule cells (DGC), though small in absolute number, have been shown to change hippocampal activity ${ }^{4}$. It has been demonstrated in adult animal models that status epilepticus (SE) increases cell proliferation in the subgranular layer of the $D G^{5,6}$. These seizure-induced newborn DGC can incorporate aberrantly in the dentate neuronal circuit, showing e.g. hilar basal dendrites, an ectopic localization and an abnormal projection of axons to both CA3 pyramidal cell regions and the inner molecular layer $(\mathrm{ML})$ of the $\mathrm{DG}^{6-8}$. Hence, seizure-induced newborn DGC may disrupt the DG filter function promoting hippocampal hyperexcitability. Blocking the process of neurogenesis after induction of SE decreases the frequency of spontaneous recurrent seizures, suggesting a pro-epileptogenic role for post-seizure born $\mathrm{DGC}^{9}$. Although it is unclear whether seizures in the developing brain change the proliferative rate of DGC (for review se $\mathrm{e}^{10}$ ), experimental neonatal FS do increase the survival of newborn $\mathrm{DGC}^{11,12}$. Moreover, newborn DGC may migrate to ectopic locations after experimental FS, a 
phenomenon that is related to hippocampal hyperactivity ${ }^{13}$. However, little is known about the post-FS born DGC that are normotopically localized in the granular cell layer.

The process of neurogenesis typically involves four milestones, i.e. proliferation, migration, differentiation and integration. Within four weeks after division, newborn DGC extend their dendrites into the $\mathrm{ML}^{14}$ and within seven weeks integration is completed ${ }^{5}$. It has been shown that granule cell maturity is correlated to dendritic length, arbor complexity and spine formation ${ }^{15,16}$. Experimental SE accelerates this integration process since, already two weeks after seizures, dendrites of normotopically located DGC extend into the middle and outer $\mathrm{ML}$, whereas in controls they only reach the inner ML. Also functional integration is increased by experimental SE since stimulation of the perforant path could elicit excitatory postsynaptic potentials in newborn DGC, which show the presence of dendritic spines, phenomena that were not seen in control animals ${ }^{17}$. Because spines are a major site of synaptic input, they could directly influence neuronal excitability.

Here, we studied the temporal maturation and structural integration of post-FS born, normotopically localized DGC. To this end, FS were evoked by treating 10-day old rats with hyperthermia $(\mathrm{HT})^{18-20}$. The next day, these animals received an intrahippocampal injection with enhanced green fluorescent protein (eGFP)-encoding retroviral particles to label dividing $D G C^{21}$. One, four and eight weeks after $\mathrm{HT}$ treatment, we analyzed co-localization of eGFP-positive cells with neurodevelopmental maturation markers and studied dendritic complexity (total dendritic length, number of branching points and Sholl analysis), overall spine density and mushroom type spine density. 
Raijmakers et al. | 6

\section{Methods}

\subsection{Animals}

In total, seventy-five male Sprague-Dawley rats were used (Harlan, Horst, The Netherlands). Six to ten pups were housed together with a foster dam and on postnatal day $(P) 22$ the dam was removed from the nest. From P36 onwards, animals were housed individually. Housing conditions were temperature-controlled at $21 \pm 1^{\circ} \mathrm{C}$, with a $12: 12 \mathrm{~h}$ light/dark cycle and animals had access to food and water ad libitum. All animal procedures were approved by the Hasselt University ethics committee for animals (ethical matrix 2012/42).

\subsection{Induction of FS}

FS were induced as described earlier ${ }^{11,18,22}$. Briefly, P10 pups were injected subcutaneously with $0.2 \mathrm{~mL} 0.9 \% \mathrm{NaCl}$ and placed in a $50 \mathrm{~cm}$ high, $10 \mathrm{~cm}$ diameter Perspex cylinder and subjected to a regulated heated air stream. Rectal temperature was measured every $2.5 \mathrm{~min}$ and maintained for $30 \mathrm{~min}$ between 39.5 and $42.5^{\circ} \mathrm{C}$ for $30 \mathrm{~min}$. Behavioral seizures, characterized by clonic contractions of fore- and hindlimbs while lying on side or back, were monitored by two observers. After HT treatment, pups were cooled down to pre-exposure body temperature by rubbing them with a water-soaked tissue and returned to the nest. As described previously, during HT treatment, a subset of animals displayed behavioural seizures $\left(\mathrm{HT}^{+}\right)$, whereas others did not $\left(\mathrm{HT}^{-}\right)^{12,20,22}$. The control group consisted of animals that were subjected to the same procedure, with the exception that they were kept normotherm (NT). 


\subsection{Production and stereotactic injection of eGFP-coding retroviral particles}

Replication-deficient enhanced GFP (eGFP)-expressing retroviral particles based on the Moloney murine leukemia virus were produced using a triple transient transfection of human embryonic kidney (HEK) 293T cells with three different plasmids containing CMVgag/pol, CMV-VSVG and CAG-eGFP (kindly donated by prof. H. van Praag, Laboratory of Neurosciences, Biomedical Research Center NIA, Baltimore, USA) ${ }^{21}$. Plasmids were transfected in HEK 293 T cells with a mixture of Lipofectamine 2000 (Invitrogen, Belgium) and Opti-MEM (Invitrogen) at $37^{\circ} \mathrm{C}$. Supernatant containing retroviral particles was collected after $72 \mathrm{~h}$, filtered through a $0.22 \mu \mathrm{m}$ filter and concentrated by two centrifugations of $19,400 \mathrm{rcf}\left(2 \mathrm{~h}\right.$ at $\left.4^{\circ} \mathrm{C}\right)$. The final pellet was resuspended in phosphate buffered saline (PBS) and stored at $-80^{\circ} \mathrm{C}$ until further use.

At P11, newborn DGC were labelled via stereotactic injection of eGFP-encoding retroviral particles. For anesthesia, animals were injected subcutaneously with $37.5 \mu \mathrm{gg} / \mathrm{kg}$ Dexdomitor $^{\circledast}$ (Orion Corporation, Finland) together with $75 \mu \mathrm{g} / \mathrm{kg}$ Temgesic $^{\circledR}$ (Reckitt Benckiser Healthcare, United Kingdom). After $10 \mathrm{~min}$, animals were intraperitoneally injected with $22.5 \mathrm{mg} / \mathrm{kg} \operatorname{Ketalar}^{\circledast}$ (Pfizer, Ireland). Animals were positioned on a temperature-controlled heating pad in a stereotactic frame with a neonatal rat adaptor (Stoelting, Illinois, USA). Next, the skin was incised and 2 drops of $2 \%$ Xylocaine $^{\circledR}$ (Recipharm Monts, France) were applied on the periost before removal. Bur holes in the skull were drilled bilaterally at anterioposterior $-3.0 \mathrm{~mm}$ and mediolateral $2.4 \mathrm{~mm}$ (relative to lambda). Each DG was infused with $2 \mu \mathrm{L}(0.25 \mu \mathrm{L} / \mathrm{min})$ using a 34G beveled needle (World Precision Instruments, United Kingdom) at dorsoventral coordinates $-3.1 \mathrm{~mm}$ and $-2.6 \mathrm{~mm}(1 \mu \mathrm{L}$ at each coordinate). After injection, the skin was closed using 3/0 VICRYL ${ }^{\mathrm{TM}}$ (Johnson and 
Johnson, Belgium). One day post-surgery, animals were injected subcutaneously with 1.5 $\mathrm{mg} / \mathrm{kg}$ Metacam $^{\circledR}$ (Labiana Life Sciences S.A., Spain) to minimize pain and suffering.

\subsection{Tissue processing}

At P17, P38 or P66, animals were injected intraperitoneally with $100 \mathrm{mg} / \mathrm{kg}$ sodium Nembutal ${ }^{\circledR}$ (Ceva Santé Animale, France) and transcardially perfused with ice-cold $0.9 \% \mathrm{NaCl}$ followed by ice-cold 4\% paraformaldehyde (PFA) in PBS. Brains were post-fixed in 4\% PFA (1h at $4^{\circ} \mathrm{C}$ ), cryoprotected in $20 \%$ sucrose in PBS (overnight at $4^{\circ} \mathrm{C}$ ) and snap frozen using liquid nitrogen.

\subsection{Immunohistochemistry}

For immunohistochemical labelling, $10 \mu \mathrm{m}$ coronal cryosections were stained with the following primary antibodies: anti-calretinin (\#MAB1568, Millipore, 1:1000), antidoublecortin (DCX) (ab18723, Abcam $^{\circledR}, 1: 2000$ for P17 tissue and 1:1000 for P38 and P66 tissue) or anti-NeuN (\#MAB377, Millipore, 1:100). After washing in PBS, cryosections were blocked with $20 \%$ normal donkey serum in PBS-0.1\% Triton (for $1 \mathrm{~h}$ at room temperature). Subsequently, sections were incubated with primary antibody diluted in PBS- $0.1 \%$ Triton containing $1 \%$ normal donkey serum (overnight at $4^{\circ} \mathrm{C}$ ). The next day, sections were incubated with secondary donkey-anti-mouse antibody conjugated to AlexaFluor-555 (A21422, Life Technologies, 1:500) or donkey-anti-rabbit conjugated to AlexaFluor-555 (A31572, Life Technologies, 1:500) (60 min at room temperature) and subsequently washed in PBS. Finally, cryosections were embedded in mounting medium containing 4',6-diamidino2-fenylindool (DAPI) (Labconsult, Belgium) and sealed with a coverglass. 
Immunohistochemical stainings were visualized using a 20x (NA of 0.50 ) or a 40x (NA of 0.75) Plan Fluor objective and a Nikon Eclipse 80i microscope connected to a Nikon digital sight camera DS-2MBWc. Cells that expressed both eGFP and one of the neuronal maturation markers were counted using NIS-elements software (Nikon Instruments Europe B.V., The Netherlands). Cell counting was done independently by two researchers who were blinded to treatment group. Strict inclusion criteria were used: 1 . only eGFP-positive cells located in the DG granule cell layer were included in the analysis, 2. each eGFP-positive cell must contain a clear DAPI positive cell nucleus and 3. clear staining of a maturation marker overlapping but not extending the eGFP signal, or clear absence of this staining must be seen. The amount of double positive cells was expressed as a \% of the total number of eGFPpositive cells that was analyzed. Per animal four cryosections were evaluated.

\subsection{Morphological analyses}

For morphological assessment, $40 \mu \mathrm{m}$ coronal cryosections were embedded in DAPIcontaining mounting medium and sealed with a coverglass. For dendritic and spine visualization, z-stacks were obtained with a Zeiss LSM510 META (Carl Zeiss, Germany) mounted on an Axiovert $200 \mathrm{M}$. For dendritic growth analysis at P17, z-stacks $(1.0 \mu \mathrm{m}$ interval) were made using a 40x water immersion objective (LD C-Apochromat 40/1.1 W Korr UV-VIS-IR, Carl Zeiss) (512x512 pixels). Dendritic length was traced using Fiji software (http://fiji.sc/Fiji; an open source image processing package based on ImageJ software (http://imagej.nih.gov/ij/)) and the number of branching points was counted manually. Dendritic arbors at P38 and P66 were visualized by acquiring z-stacks ( $0.5 \mu \mathrm{m}$ interval) using a 20x Plan-Apochromat (NA of 0.75) (1024×1024 pixels). Stacks of images were compressed along the z-axis to two-dimensional images and whole dendritic arbors were traced using Fiji 
Raijmakers et al. | 10

and analyzed for dendritic length and number of branching points. Next, dendritic complexity was further determined using Sholl analysis by counting the number of dendrite intersections for a series of concentric circles at $5 \mu \mathrm{m}$ intervals around the soma. Truncated cells were excluded from the analysis. An average of 3 cells/animal were analyzed. Spines were imaged by obtaining z-stacks (0.25 $\mu \mathrm{m}$ interval) using a 100x/1.46 alpha PlanApochromat objective ( $1024 \times 500$ pixels) and a scan zoom of 3 . To determine spine density, we divided the $M L$ in two parts as described by Murphy et $\mathrm{al}^{23}$, i.e. the innermost $17 \%$ which is defined as the inner $\mathrm{ML}(\mathrm{IML})$ and the outer $83 \%$ containing the middle and outer $\mathrm{ML}$ $(M M L+O M L)$. Length of dendritic segments was measured automatically from 3D images using Fiji. The number of spines on dendritic segments were counted manually on the 3D images using Fiji software by two researchers who were blinded to treatment (average of 5 segments per animal). Strict inclusion criteria were used to include spines in analyses: 1. spines had to be connected to the dendritic branch. 2. no adjacent dendritic segments were present in the analyzed confocal photograph. Spine head surface area was calculated $\left(\pi^{*} D_{\text {major }} * D_{\text {minor }} / 4\right)$ and mushroom type spines were identified when surface area $>0.4 \mu m^{2}$ $16,24$.

\subsection{Statistics}

As data were not normally distributed, statistical significance was assessed by the nonparametric Kruskal-Wallis test, and in case of significance, followed by post-hoc Dunn's comparison of columns, using GraphPad Prism 5. P-values $<0.05$ were considered as significant. In case of significance in Sholl analysis, post-hoc individual student t-tests were performed (GraphPad Software, Inc., La Jolla, USA). To avoid type I errors due to multiple ttests, only p-values $<0.05$ of three or more consecutive points were considered as 
significantly different ${ }^{25}$. Outliers, assessed by descriptive statistics using SPSS22 (IBM Corp., IBM SPSS Statistics Version 22.0, NY, USA), were excluded from the data. Values are expressed as median with interquartile range (IQR) and whiskers (5-95\% interval) or as mean \pm standard error of the mean (SEM). The number of animals is represented by $n$.

\section{Results}

\subsection{FS do not change the temporal expression of neurodevelopmental markers in newborn DGC}

Previous research using the HT rat model of FS indicated that the majority of post-FS born DGC were differentiated into mature neurons in the DG granule layer at $P 66^{26}$. We determined the pace of this maturation by analyzing eGFP-labelled post-FS born DGC at three time points (P17, P38 and P66) by double labelling with different neurodevelopmental markers (DCX, calretinin and NeuN). The immature neuronal cell marker DCX was ubiquitously present in the DG granule cell layer of P17 NT controls. This implies that the vast majority of eGFP-labelled DGC expressed DCX (Fig. 1 A-C), while quantification revealed a substantial decline in the proportion of DCX/eGFP double-labelled DGCs to $9 \%$ at P38 and 2\% at P66 (Fig. $1 \mathrm{D}-\mathrm{F}, \mathrm{M}$ ). These percentages were similar in $\mathrm{HT}^{+}$and $\mathrm{HT}^{-}$rats (Fig. 1M). Labelling of early granule cells in the post-mitotic stage was performed by using calretinin. At P17, no calretinin-positive cells could be detected in the DG in none of the three experimental groups (data not shown). At P38 and P66, calretinin expression was present in the DG of NT rats, yet no eGFP-positive DGC co-labelled with calretinin in all three experimental groups (Fig. 1 G-I). In contrast, a large proportion of eGFP-labelled cells coexpressed the mature neuronal marker NeuN, i.e. $79 \%$ at $\mathrm{P} 38$ and $86 \%$ at $\mathrm{P} 66$ in NT rats. These numbers were not altered in $\mathrm{HT}^{+}$and $\mathrm{HT}^{-}$rats compared to NT controls (Fig. $1 \mathrm{~J}-\mathrm{L}, \mathrm{N}$ ). 
Raijmakers et al. | 12

\subsection{FS increase dendritic complexity of newborn DGC}

To investigate how FS-induced newborn granule cells incorporate into the existing hippocampal network, dendritic complexity of eGFP-labeled post-FS born DGC was determined by scoring their total dendritic length and their number of branching points at P17, P38 and P66.

At P17 (Fig. 2), eGFP-labelled DGC in $\mathrm{HT}^{+}$rats showed $66 \%$ longer dendrites $(\mathrm{p}<0.001)$ compared to NT controls and $97 \%$ longer dendrites $(p<0.001)$ compared to $\mathrm{HT}^{-}$rats (Fig. 2D). The number of branching points in $\mathrm{HT}^{+}$rats was similar to NT controls and significantly increased compared to $\mathrm{HT}^{-}$rats $(\mathrm{p}<0.05)$ (Fig. 2E). Dendrites reached the outer part of the $M L$ within four weeks after cell division and the furthest dendritic extension (in NT controls: $258 \mu \mathrm{m}$ at P38 and $275 \mu \mathrm{m}$ at P66) was comparable in all experimental groups. Neither seizures nor HT treatment affected dendritic length or the number of branching points at P38 (Fig. 3K,L) or P66 (Fig. 4K,L). Next, we performed a Sholl analysis to express dendritic complexity in function of the distance to the cell soma, i.e. the specific location in the ML. At P38, the number of dendrite crossings with consecutive Sholl circles located at 130-155 $\mu \mathrm{m}$ from cell soma was significantly increased in $\mathrm{HT}^{+}$rats compared to $\mathrm{NT}$ and $\mathrm{HT}^{-}$rats $(\mathrm{p}<0.01$; Fig. 3J). At P66, Sholl analysis revealed a significantly enhanced dendritic complexity in $\mathrm{HT}^{+}$ and $\mathrm{HT}^{-}$rats compared to NT controls ( $\mathrm{p}<0.01$; Fig. 4J). More specifically, dendritic complexity in $\mathrm{HT}^{+}$animals was increased at 105-185 and 195-215 $\mu \mathrm{m}$ from cell soma. In $\mathrm{HT}^{-}$ animals significant differences were situated 100-150 and 160-215 $\mu \mathrm{m}$ from cell soma.

\subsection{Newborn DGC display more mushroom spines eight weeks after FS}

We determined dendritic spine density to estimate excitatory synapse density, though only at P38 (Fig. 3 G-I,M) and P66 (Fig. 4 G-I,M) since spines were not present in eGFP-positive 
DGC at P17 in any of the experimental groups (data not shown). This is consistent with previous observations ${ }^{16}$.

Overall dendritic spine density was similar in the IML as well as in the $\mathrm{MML}+\mathrm{OML}$ of $\mathrm{HT}^{+}$and $\mathrm{HT}^{-}$rats compared to NT controls at P38 (Fig. $3 \mathrm{M}$ ) and P66 (Fig. $4 \mathrm{M}$ ). Furthermore, spine head surface analyses at P38 revealed comparable numbers of mushroom spines in the IML as well as the MML+OML of all experimental groups (Fig. $3 \mathrm{~N}$ ). However, at P66 the amount of mushroom spines was significantly increased in the $\mathrm{MML}+\mathrm{OML}$ in $\mathrm{HT}^{+}$animals compared to NT controls $(p<0.05)$, whereas in the IML the density of this spine type was unaltered (Fig. $4 N)$.

\section{Discussion}

It is well known that complex FS induce a decreased seizure threshold leading to a hyperexcitable state of the hippocampal network ${ }^{3}$. As the hippocampal DG is a site where neurogenesis occurs and since cell survival of post-FS born granule cells is increased ${ }^{11,12}$, we aimed to elucidate how these newborn granule cells incorporate into the existing hippocampal network. In the present study, the neuronal maturation stage and the structural integration of these newborn cells were investigated at different time points (one week, four weeks and eight weeks) following experimental FS. The maturation rate was unaltered, however, the structural integration was accelerated after experimental FS. This increased integration was demonstrated by an increased dendritic length at one week and an enhanced dendritic complexity at four and eight weeks, when cells show a fully mature phenotype.

Hippocampal neurogenesis has been shown to play a significant role in the process of epileptogenesis ${ }^{5,6,12,26}$. Since neurogenesis in the DG is particularly active in rats around the 
Raijmakers et al. | 14

age of 10 days, changes in this process during the neonatal period may significantly affect the filtering function of the $D G^{27}$. There are strong indications from the experimental $F S$ model that newborn DGC behave abnormally, as a subset of newly born cells migrate incorrectly to the hilar $\mathrm{DG}^{13}$. Several animal FS and TLE models have shown that the occurrence of spontaneous seizures is associated with abnormal granule cell localization ${ }^{13,28,29}$. However, it also has been stated that this phenomenon alone is not sufficient to cause epilepsy ${ }^{13}$. The vast majority of DGC born after FS migrates correctly to the middle and upper layer of the granular $\mathrm{DG}^{13}$. So far, little is known about the role of these normotopically localized post-FS born DGC in the generation of an epileptogenic hippocampal network. Consistent with previous data ${ }^{26}$, we show here that the majority of normotopic post-FS born DGC develop a mature neuronal phenotype. Since analysis of the neuronal maturation rate might gain insight into the role of newborn DCG during the process of epileptogenesis, we additionally examined the expression of neurodevelopmental markers DCX, calretinin and NeuN at different time points after experimental FS. It has been shown by others that one week after kainic-acid induced SE, the majority of newborn granule cells co-localize with the early neuronal marker DCX in both treated and control animals $^{30}$. In line with this, we also see that one week after experimental FS, newborn granule cells co-localize mainly with DCX. This co-localization decreases over time and the majority of cells express the mature neuronal marker NeuN after four and eight weeks. The marker for early granule cells in the post-mitotic stage was not expressed by FS-induced newborn cells which is most likely due to the limited time window of calretinin expression ${ }^{31}$. We did not find any differences between animals that experienced FS and controls and therefore we conclude that, at least based on the expression of neurodevelopmental 
Raijmakers et al. | 15

markers over time, newborn cells mature into adult neurons at the same rate in all experimental groups.

Although the rate of maturation is unchanged in FS animals versus controls, the structural integration of these cells seems to be altered. Here, we show that dendritic growth is increased in newborn normotopic DGC one week following FS. Moreover, we demonstrate that in FS rats the enhanced dendritic complexity sustains for up to eight weeks in the $83 \%$ outer part of the $\mathrm{ML}$, which can be subdivided equally into the $\mathrm{MML}$ and $\mathrm{OML}$ according to Murphy et $\mathrm{al}^{23}$. Enhanced dendritic complexity is mainly confined to the MML at P38 while at P66 the area with increased dendritic complexity is drastically extended involving the OML also. Increased dendritic length and structural integration has already been described in other adult and neonatal models for seizures ${ }^{17,32,33}$. In line with our results, OverstreetWadiche et al. (2006) reported that newborn DGC extended their dendrites in the OML two weeks post-SE, whereas dendrites of control cells were restricted to the $\mathrm{IML}^{17}$. Importantly, these findings were obtained from a pro-opiomelanocortin-eGFP transgenic mice in which newborn DGC expressed eGFP only transiently, i.e. during the first weeks after cell birth. In contrast, we pulse-chased only those DGC that were born after FS and could demonstrate that their changes in dendritic structure are persistent up to at least eight weeks. In contrast with our findings, Koyama et al. described a comparable dendritic complexity in FS animals versus controls ${ }^{13}$. Although it is not clear whether these cells reflect normotopic or ectopic cells, it is worth noting that, in this study, newborn cells were labelled prior to, instead of after experimental FS induction. As reported by Kron et al., the developmental stage of DGC at the time of an insult determines the type of morphological alteration ${ }^{7}$ and in our study we have labelled cells that are dividing shortly after experimental FS. 
Raijmakers et al. | 16

In line with previous studies ${ }^{12,18,20,26}$, we also describe an experimental group consisting of HT-treated animals without behavioral seizures. At P17 and P38, dendritic complexity in $\mathrm{HT}^{-}$ rats resembles that of NT rats suggesting that changes in dendritic complexity are a seizurespecific effect. In contrast, at eight weeks after HT-treatment the dendritic complexity in $\mathrm{HT}^{-}$ rats is comparable to $\mathrm{HT}^{+}$rats. Yet, it has to be stressed that we did not confirm seizure activity with an electroencephalogram. Hence, it is possible that $\mathrm{HT}^{-}$rats experienced subclinical seizures during or after HT treatment ${ }^{12}$.

Previously, we have shown that post-FS born granule cells in $\mathrm{HT}^{+}$animals express more often the $\mathrm{GABA}_{\mathrm{A}}$ receptor compared to eight weeks controls after $\mathrm{HT}$ treatment ${ }^{12}$. $\mathrm{GABA}_{\mathrm{A}}$ receptors may display excitatory instead of inhibitory effects in newborn cells and epileptogenic conditions ${ }^{34}$. Moreover, depolarizing GABA-ergic input can accelerate maturation and synaptic integration of immature neurons ${ }^{35}$. The increased $G A B A_{A}$ receptor expression combined with the potential depolarizing GABA effect may underlie the enhanced maturation processes of newly formed cells after experimental FS. In line with this, it was shown that $\mathrm{GABA}_{\mathrm{A}}$ receptor signaling mediated the correct migration of newborn granule cells and that this process is disrupted after experimental FS induction presumably by excitatory GABA effects ${ }^{13}$.

The higher degree of dendritic arborization in the MML and OML suggests an increased excitatory input coming from respectively the medial and lateral entorhinal cortex ${ }^{23}$. This hypothesis is in line with previous results from the pilocarpine model in which induction of SE reduces inhibitory synaptic input in layer II of the medial entorhinal cortical neurons causing excessive excitatory synaptic input into the $\mathrm{DG}^{36}$. In addition, increased VGLUT-1 immunoreactivity in the $M L$ and an increase in the excitability ratio in the DG was observed 
in the experimental FS model suggesting augmented excitatory input to the DG ${ }^{37}$. As dendritic spines are the major postsynaptic sites for excitatory synaptic input, spine density can be used as a measure for excitatory synapse density. Although we did not find any influence of FS on overall spine density, we showed an increase in the amount of mushroom type spines in the MML+OML at P66. Mushroom spines contain a larger postsynaptic density which has a direct positive correlation with synaptic strength ${ }^{38}$. An increase in mushroom type spines therefore further indicates a more mature phenotype of the newborn DGC in $\mathrm{HT}^{+}$animals compared to controls, presumably caused by an increased input from the entorhinal cortex ${ }^{39}$

In conclusion, we show that experimental FS enhance structural integration of newborn granule cells (i.e., increase in dendritic complexity and mushroom type spines) and thereby possibly increase connectivity with the perforant pathway, which supports the idea that these cells contribute to the hyperexcitable state seen after FS.

\section{Acknowledgements}

We greatly appreciate the kindness of $\mathrm{H}$. van Praag for providing plasmids used for virus production. Furthermore we would like to thank Rik Paesen for his help with the confocal microscope and Petra Bex and Rosette Beenaerts for their assistance with the virus production and immunohistochemistry. This research was financially supported by a 'Bijzonder Onderzoeksfonds' grant from Hasselt University. Nick Smisdom was supported by a post-doctoral scholarship of the Research Foundation - Flanders (FWO-Vlaanderen).

\section{Disclosure of conflicts of interest}


None of the authors has any conflict of interest to disclose. The authors confirm that they have read the Journal's position on issues involved in ethical publication and affirm that their report is consistent with those guidelines.

\section{References}

1 Hauser WA. The prevalence and incidence of convulsive disorders in children. Epilepsia 1994;35:S1-S6.

2 Berg AT, Shinnar S. Complex febrile seizures. Epilepsia 1996;37:126-133.

3 Dubé $\mathrm{CM}$, Brewster AL, Richichi $\mathrm{C}$, et al. Fever, febrile seizures and epilepsy. Trends in Neurosciences 2007;30:490-496.

4 Lacefield CO, Itskov V, Reardon $\mathrm{T}$, et al. Effects of adult-generated granule cells on coordinated network activity in the dentate gyrus. Hippocampus 2012;22:106-116.

5 Jessberger $\mathrm{S}$, Kempermann G. Adult-born hippocampal neurons mature into activitydependent responsiveness. Eur J Neurosci 2003;18:2707-2712.

6 Parent JM, Yu TW, Leibowitz RT, et al. Dentate granule cell neurogenesis is increased by seizures and contributes to aberrant network reorganization in the adult rat hippocampus. $J$ Neurosci 1997;17:3727-3738.

$7 \quad$ Kron MM, Zhang H, Parent JM. The developmental stage of dentate granule cells dictates their contribution to seizure-induced plasticity. J Neurosci 2010;30:2051-2059.

8 Parent JM, Tada E, Fike JR, et al. Inhibition of dentate granule cell neurogenesis with brain irradiation does not prevent seizure-induced mossy fiber synaptic reorganization in the rat. $J$ Neurosci 1999;19:4508-4519.

9 Jung KH, Chu K, Kim M, et al. Continuous cytosine-b-D-arabinofuranoside infusion reduces ectopic granule cells in adult rat hippocampus with attenuation of spontaneous recurrent seizures following pilocarpine-induced status epilepticus. Eur J Neurosci 2004;19:3219-3226.

10 Porter BE. Neurogenesis and epilepsy in the developing brain. Epilepsia 2008;49:50-54.

11 Lemmens EM, Lubbers T, Schijns OEM, et al. Gender differences in febrile seizure-induced proliferation and survival in the rat dentate gyrus. Epilepsia 2005;46:1603-1612.

12 Swijsen A, Brône B, Rigo JM, et al. Long-lasting enhancement of GABAA receptor expression in newborn dentate granule cells after early-life febrile seizures. Dev Neurobiol 2012;72:1516-1527.

13 Koyama R, Tao K, Sasaki T, et al. GABAergic excitation after febrile seizures induces ectopic granule cells and adult epilepsy. Nat Med 2012;18:1271-1278.

14 Deshpande A, Bergami M, Ghanem A, et al. Retrograde monosynaptic tracing reveals the temporal evolution of inputs onto new neurons in the adult dentate gyrus and olfactory bulb. Proc Natl Acad Sci U S A 2013;110:E1152-E1161.

15 Wang S, Scott BW, Wojtowicz JM. Heterogenous properties of dentate granule neurons in the adult rat. $J$ Neurobiol 2000;42:248-257.

16 Zhao C, Teng EM, Summers RG, et al. Distinct morphological stages of dentate granule neuron maturation in the adult mouse hippocampus. J Neurosci 2006;26:3-11.

17 Overstreet-Wadiche LS, Bromberg DA, Bensen AL, et al. Seizures accelerate functional integration of adult-generated granule cells. J Neurosci 2006;26:4095-4103.

18 Baram TZ, Gerth A, Schultz L. Febrile seizures: an appropriate-aged model suitable for longterm studies. Brain Res Dev Brain Res 1997;98:265-270.

19 Dubé C, Richichi C, Bender RA, et al. Temporal lobe epilepsy after experimental prolonged febrile seizures: prospective analysis. Brain 2006;129:911-922. 
Swijsen A, Avila A, Brône B, et al. Experimental early-life febrile seizures induce changes in GABAAR-mediated neurotransmission in the dentate gyrus. Epilepsia 2012;53:1968-1977.

21 van Praag $\mathrm{H}$, Schinder $A F$, Christie $B R$, et al. Functional neurogenesis in the adult hippocampus. Nature 2002;415:1030-1034.

22 Swijsen A, Nelissen K, Janssen D, et al. Validation of reference genes for quantitative realtime PCR studies in the dentate gyrus after experimental febrile seizures. BMC Res Notes 2012;5:685.

23 Murphy BL, Pun RY, Yin $\mathrm{H}$, et al. Heterogeneous integration of adult-generated granule cells into the epileptic brain. J Neurosci 2011;31:105-117.

24 Zhao $C$, Jou J, Wolff $\amalg$, et al. Spine morphogenesis in newborn granule cells is differentially regulated in the outer and middle molecular layers. J Comp Neurol. 2014;522:2756-2766.

25 Smith KC, Ehlinger DG, Smith RF. Adolescent nicotine alters dendritic morphology in the bed nucleus of the stria terminalis. Neurosci Lett 2015;590:111-115.

26 Lemmens EMP, Schijns OEMG, Beuls EAM, et al. Cytogenesis in the dentate gyrus after neonatal hyperthermia-induced seizures: What becomes of surviving cells? Epilepsia 2008;49:853-860.

27 Overstreet LS, Hentges ST, Bumaschny VF, et al. A transgenic marker for newly born granule cells in dentate gyrus. J Neurosci 2004;24:3251-3259.

28 Parent JM, Murphy GG. Mechanisms and functional significance of aberrant seizure-induced hippocampal neurogenesis. Epilepsia 2008;49 Suppl 5:19-25.

29 Scharfman H, Goodman J, McCloskey D. Ectopic granule cells of the rat dentate gyrus. Dev Neurosci 2007;29:14-27.

30 Laurén HB, Ruohonen S, Kukko-Lukjanov TK, et al. Status epilepticus alters neurogenesis and decreases the number of GABAergic neurons in the septal dentate gyrus of 9-day-old rats at the early phase of epileptogenesis. Brain Res 2013;1516:33-44.

31 Kempermann G, Jessberger S, Steiner B, et al. Milestones of neuronal development in the adult hippocampus. Trends Neurosci 2004;27:447-452.

32 Overstreet-Wadiche LS, Bensen AL, Westbrook GL. Delayed development of adult-generated granule cells in dentate gyrus. J Neurosci 2006;26:2326-2334.

33 Pugh $\mathrm{P}$, Adlaf $\mathrm{E}$, Zhao CS, et al. Enhanced integration of newborn neurons after neonatal insults. Front Neurosci 2011;5:45.

34 Kahle KT, Staley KJ, Nahed BV, et al. Roles of the cation-chloride cotransporters in neurological disease. Nat Clin Pract Neuro 2008;4:490-503.

35 Ge S, Goh EL, Sailor KA, et al. GABA regulates synaptic integration of newly generated neurons in the adult brain. Nature 2006;439:589-593.

36 Kobayashi M, Wen X, Buckmaster PS. Reduced inhibition and increased output of layer II neurons in the medial entorhinal cortex in a model of temporal lobe epilepsy. $J$ Neurosci 2003;23:8471-8479.

37 Kwak SE, Kim JE, Kim SC, et al. Hyperthermic seizure induces persistent alteration in excitability of the dentate gyrus in immature rats. Brain Res 2008;1216:1-15.

38 Bosch M, Hayashi Y. Structural plasticity of dendritic spines. Curr Opin Neurobiol 2012;22:383-388.

39 Gao F, Song X, Zhu D, et al. Dendritic morphology, synaptic transmission, and activity of mature granule cells born following pilocarpine-induced status epilepticus in the rat. Front. Cell. Neurosci. 2015;9. 
Raijmakers et al. | 20

\section{Figure legends}

Figure 1: HT-induced seizures do not alter the temporal expression of neurodevelopmental markers in newborn DGC. Typical photomicrographs of DCX immunoreactive DGC (A), postHT born DGC labelled with eGFP (B) and colocalization of DCX and eGFP (C) in a $\mathrm{HT}^{-}$animal at P17. Typical photomicrographs of DCX (D), calretinin (G) and NeuN (J) immunoreactive DGC, post-HT born DGC labelled with eGFP (E, H and K) and colocalization of the respective maturation markers with eGFP $(\mathrm{F}, \mathrm{I}$ and $\mathrm{L})$ in a $\mathrm{HT}^{+}$animal at P38. Arrows indicate examples of double-positive cells. Scale bars: A-F, J-L $20 \mu \mathrm{m}, \mathrm{G}-\mathrm{I} 50 \mu \mathrm{m}$. Quantitative analysis of the amount of DCX/eGFP (M) and NeuN/eGFP (N) double-labelled DGC at P38 and P66. Data are represented as median (black bar), 25-75 percentile (box) and 5-95 percentile (whiskers); P38: NT $n=5, H T^{+} n=7, H T^{-} n=5$; P66: NT $n=6, H T^{+} n=5, H T^{-} n=8$.

Figure 2: At one week of development, post-seizure born DGC show longer dendrites. Photomicrographs of hippocampal DGC, born and eGFP labeled in animals $24 \mathrm{~h}$ after NT (A), $\mathrm{HT}^{+}$(B) or $\mathrm{HT}^{-}$(C) treatment, and visualized at P17. Nuclei stained blue with DAPI. ML= molecular layer, $\mathrm{GCL}=$ granular cell layer, $\mathrm{H}=$ hilus, scale bar= $50 \mu \mathrm{m}$. Quantification of the dendritic length (D) and number of branching points (E) of eGFP labelled DGC. Data are represented as median (black bar), 25-75 percentile (box) and 5-95 percentile (whiskers). ${ }^{*} p<0.05 ;{ }^{* *} p<0.001 ; N T n=4, H^{+} n=5, H T^{-} n=5$.

Figure 3: At four weeks of development, post-seizure born DGC show increased dendritic complexity and unaltered spine density. Photomicrographs of hippocampal DGC, born and eGFP labeled $24 \mathrm{~h}$ after $\mathrm{NT}(\mathrm{A}), \mathrm{HT}^{+}$(B) or $\mathrm{HT}^{-}(\mathrm{C})$ treatment and visualized at P38. Examples of dendritic traces (D-F) that were acquired from respective photomicrographs (A-C). Representative pictures of dendritic spines in the IML and MML+OML after NT (G), $\mathrm{HT}^{+}(\mathrm{H})$ or $\mathrm{HT}^{-}$(I) treatment. Arrowheads indicate mushroom type spines. Sholl analysis of dendritic complexity of eGFP labelled DGC (J). Quantification of total dendritic length (K) and number of branching points (L) of eGFP labelled DGC. Quantitative analysis of the overall spine density $(M)$ and mushroom type spine density $(N)$ in dendritic segments located in the IML and the MML+OML. Data are represented as median (black bar), 25-75 percentile (box) and 5-95 percentile (whiskers; K, L, M, N) or as mean \pm SEM (J). * $=\mathrm{HT}^{+}$vs NT: $p<0.01 ; \mathrm{HT}^{+} \mathrm{n}=4$, 
$\mathrm{HT}^{-} \mathrm{n}=4, \mathrm{NT} \mathrm{n}=4$. $\mathrm{GCL}=$ granular cell layer, $\mathrm{IML}=$ inner molecular layer, $\mathrm{MML}=$ middle molecular layer, $\mathrm{OML}=$ outer molecular layer. Scale bars: $\mathrm{A}-\mathrm{C} 50 \mu \mathrm{m}, \mathrm{G}-\mathrm{I} 5 \mu \mathrm{m}$.

Figure 4: At eight weeks of development, post-seizure born DGC show increased dendritic complexity and altered spine morphology. Photomicrographs of hippocampal DGC, born and eGFP labeled $24 \mathrm{~h}$ after $\mathrm{NT}(\mathrm{A}), \mathrm{HT}^{+}$(B) or $\mathrm{HT}^{-}$(C) treatment and visualized at P66. Examples of dendritic traces (D-F) that were acquired from respective photomicrographs (AC). Representative pictures of dendritic spines in the IML and $M M L+O M L$ after $N T(G), H T^{+}$ (H) or $\mathrm{HT}^{-}$(I) treatment. Arrowheads indicate mushroom type spines. Sholl analysis of dendritic complexity of eGFP labelled DGC (J). Quantification of total dendritic length (K) and number of branching points (L) of eGFP labelled DGC. Quantitative analysis of the overall spine density in dendritic segments located in the IML and the MML+OML (M). Quantitative analysis of the mushroom type spine density in dendritic segments located in the IML and the MML+OML (N). Data are represented as median (black bar), 25-75 percentile (box) and 5-95 percentile (whiskers; $\mathrm{K}, \mathrm{L}, \mathrm{M}, \mathrm{N}$ ) or as mean $\pm \mathrm{SEM}(\mathrm{J}){ }^{*}=\mathrm{HT}^{+}$vs NT, $\mathrm{p}<0.01 ; \neq=\mathrm{HT}^{+}$vs $\mathrm{HT}^{-}, \mathrm{p}<0.01 ;+=\mathrm{HT}^{+}$vs NT, $\mathrm{p}<0.05 ; \mathrm{HT}^{+} \mathrm{n}=4, \mathrm{HT}^{-} \mathrm{n}=4, \mathrm{NT} \mathrm{n}=4 . \mathrm{GCL}=$ granular cell layer, $\mathrm{IML}=$ inner molecular layer, $\mathrm{MML}=$ middle molecular layer, $\mathrm{OML}=$ outer molecular layer. Scale bars: A-C $50 \mu \mathrm{m}, \mathrm{G}-\mathrm{I} 5 \mu \mathrm{m}$.

\section{Figures}



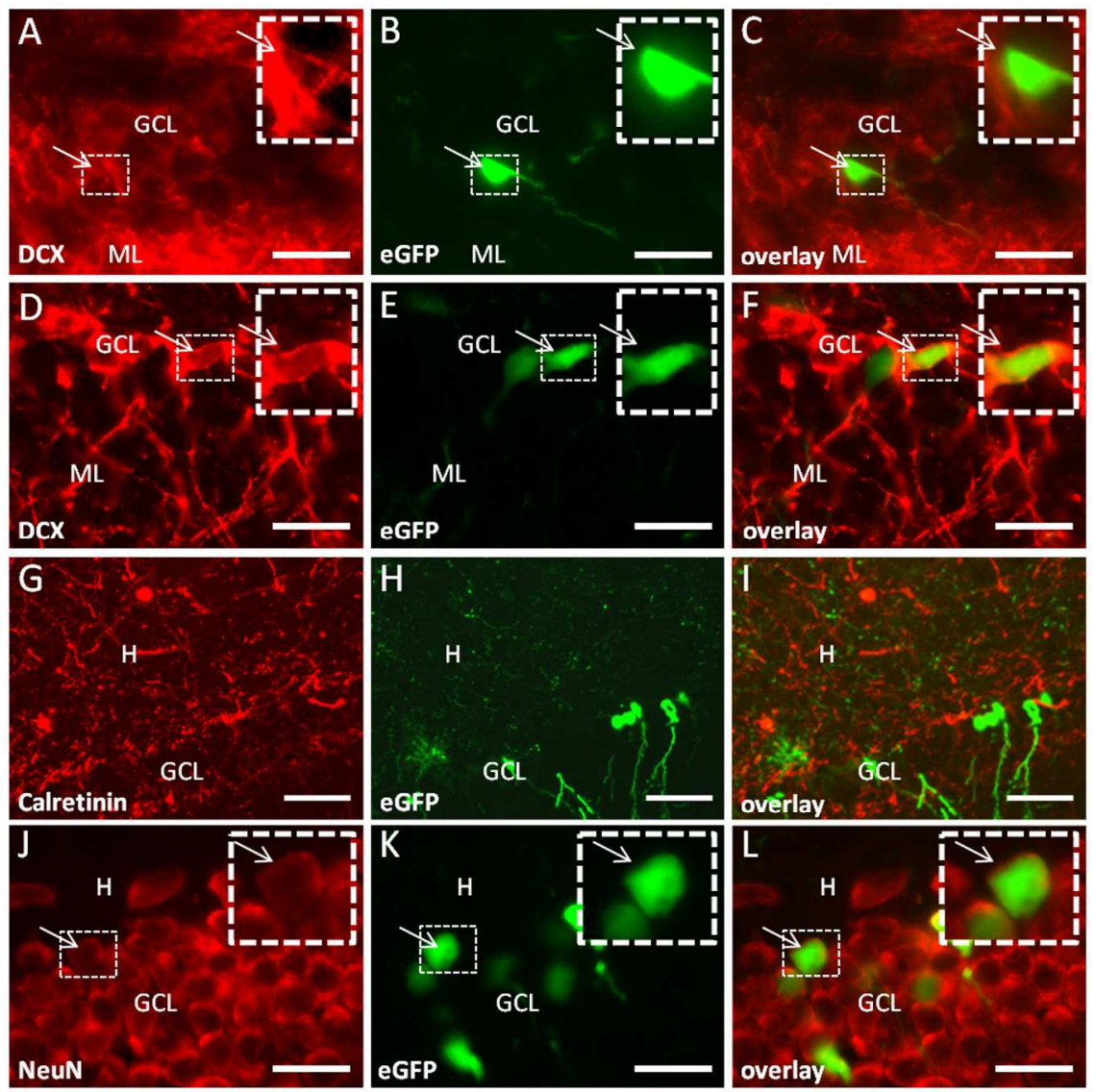

M

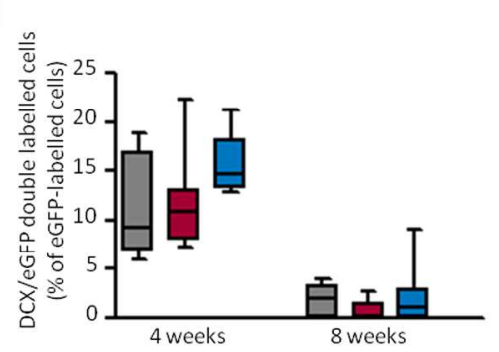

N

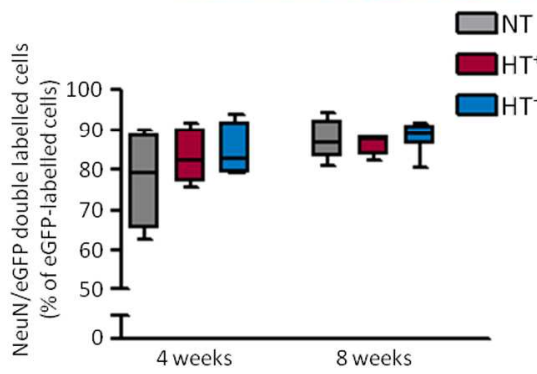

Figure 1: HT-induced seizures do not alter the temporal expression of neurodevelopmental markers in newborn DGC. Typical photomicrographs of DCX immunoreactive DGC (A), post-HT born DGC labelled with eGFP (B) and colocalization of DCX and eGFP (C) in a $\mathrm{HT}^{\top}$ animal at P17. Typical photomicrographs of DCX (D), calretinin (G) and NeuN (J) immunoreactive DGC, post-HT born DGC labelled with eGFP $(E, H$ and $K)$ and colocalization of the respective maturation markers with eGFP $(F, I$ and $L)$ in a $\mathrm{HT}^{+}$animal at P38. Arrows indicate examples of double-positive cells. Scale bars: A-F, J-L $20 \mu \mathrm{m}, \mathrm{G}-\mathrm{I} 50$ $\mu \mathrm{m}$. Quantitative analysis of the amount of DCX/eGFP (M) and NeuN/eGFP (N) double-labelled DGC at P38 and P66. Data are represented as median (black bar), 25-75 percentile (box) and 5-95 percentile (whiskers); P38: NT $n=5, \mathrm{HT}^{+} \mathrm{n}=7, \mathrm{HT}^{-} \mathrm{n}=5$; P66: NT $\mathrm{n}=6, \mathrm{HT}^{+} \mathrm{n}=5, \mathrm{HT}^{-} \mathrm{n}=8$. 

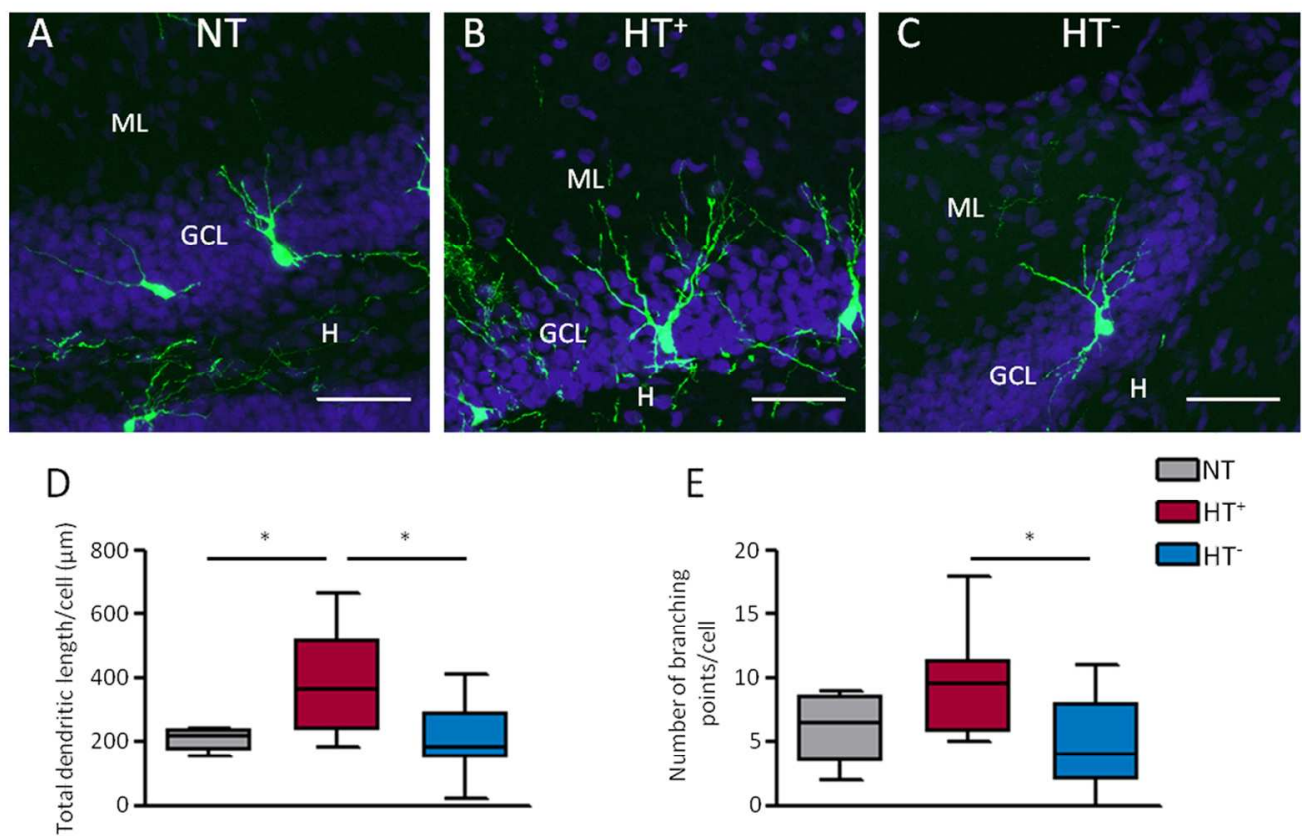

Figure 2: At one week of development, post-seizure born DGC show longer dendrites.

Photomicrographs of hippocampal DGC, born and eGFP labeled in animals $24 \mathrm{~h}$ after $\mathrm{NT}(\mathrm{A}), \mathrm{HT}^{+}(\mathrm{B})$ or $\mathrm{HT}^{-}$ (C) treatment, and visualized at P17. Nuclei stained blue with DAPI. ML= molecular layer, GCL= granular cell layer, $\mathrm{H}=$ hilus, scale bar $=50 \mu \mathrm{m}$. Quantification of the dendritic length (D) and number of branching points (E) of eGFP labelled DGC. Data are represented as median (black bar), 25-75 percentile (box) and 595 percentile (whiskers). ${ }^{*} \mathrm{p}<0.05 ; * * \mathrm{p}<0.001 ; \mathrm{NT} \mathrm{n}=4, \mathrm{HT}^{+} \mathrm{n}=5, \mathrm{HT}^{-} \mathrm{n}=5$. 

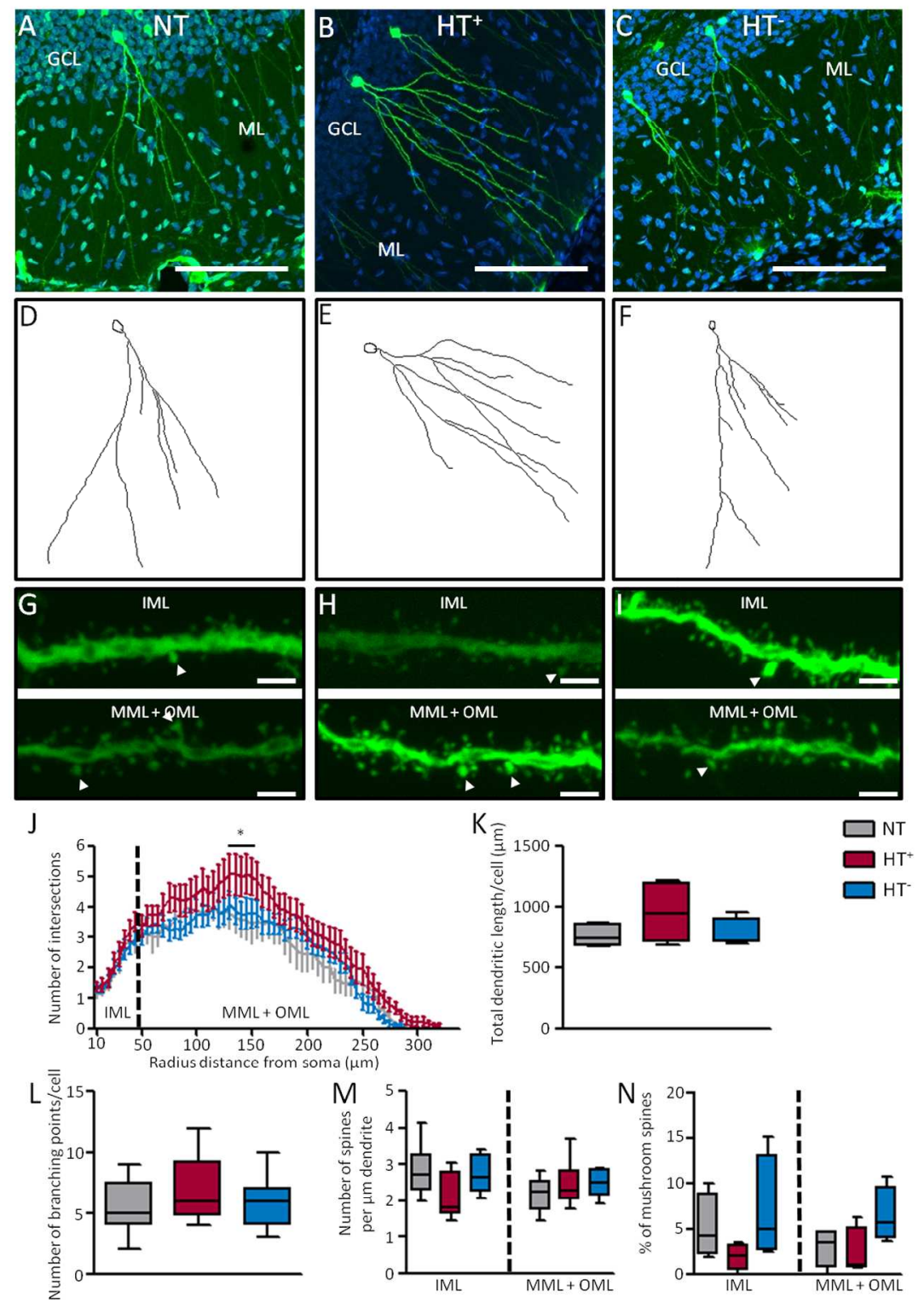

Figure 3: At four weeks of development, post-seizure born DGC show increased dendritic complexity and unaltered spine density. Photomicrographs of hippocampal DGC, born and eGFP labeled $24 \mathrm{~h}$ after $\mathrm{NT}(\mathrm{A}), \mathrm{HT}^{+}$(B) or $\mathrm{HT}^{-}$(C) treatment and visualized at P38. Examples of dendritic traces (D-F) that were acquired from respective photomicrographs $(A-C)$. Representative pictures of dendritic spines in the IML and MML+OML after NT (G), $\mathrm{HT}^{+}(\mathrm{H})$ or $\mathrm{HT}^{-}(\mathrm{I})$ treatment. Arrowheads indicate mushroom type spines. Sholl analysis of dendritic complexity of eGFP labelled DGC (J). Quantification of total dendritic length (K) and number of branching points (L) of eGFP labelled DGC. Quantitative analysis of the overall spine density (M) and mushroom type spine density $(\mathrm{N})$ in dendritic segments located in the IML and the

MML+OML. Data are represented as median (black bar), 25-75 percentile (box) and 5-95 percentile (whiskers; K, L, M, N) or as mean \pm SEM (J). $*=H T^{+}$vs NT: $p<0.01 ; \mathrm{HT}^{+} n=4, \mathrm{HT}^{-} \mathrm{n}=4, \mathrm{NT} n=4 . \mathrm{GCL}=$ granular cell layer, IML = inner molecular layer, $\mathrm{MML}=$ middle molecular layer, $\mathrm{OML}=$ outer molecular layer. Scale bars: A-C $50 \mu \mathrm{m}$, G-I $5 \mu \mathrm{m}$. $267 \times 383 \mathrm{~mm}(300 \times 300 \mathrm{DPI})$ 
Page 25 of 25

Epilepsia

1
2
3
4
5
6
7
8
9
10
11
12
13
14
15
16
17
18
19
20
21
22
23
24
25
26
27
28
29
30
31
32
33
34
35
36
37
38
39
40
41
42
43
44
45
46
47
48
49
50
51
52
53
54
55
56
57
58
59
6 

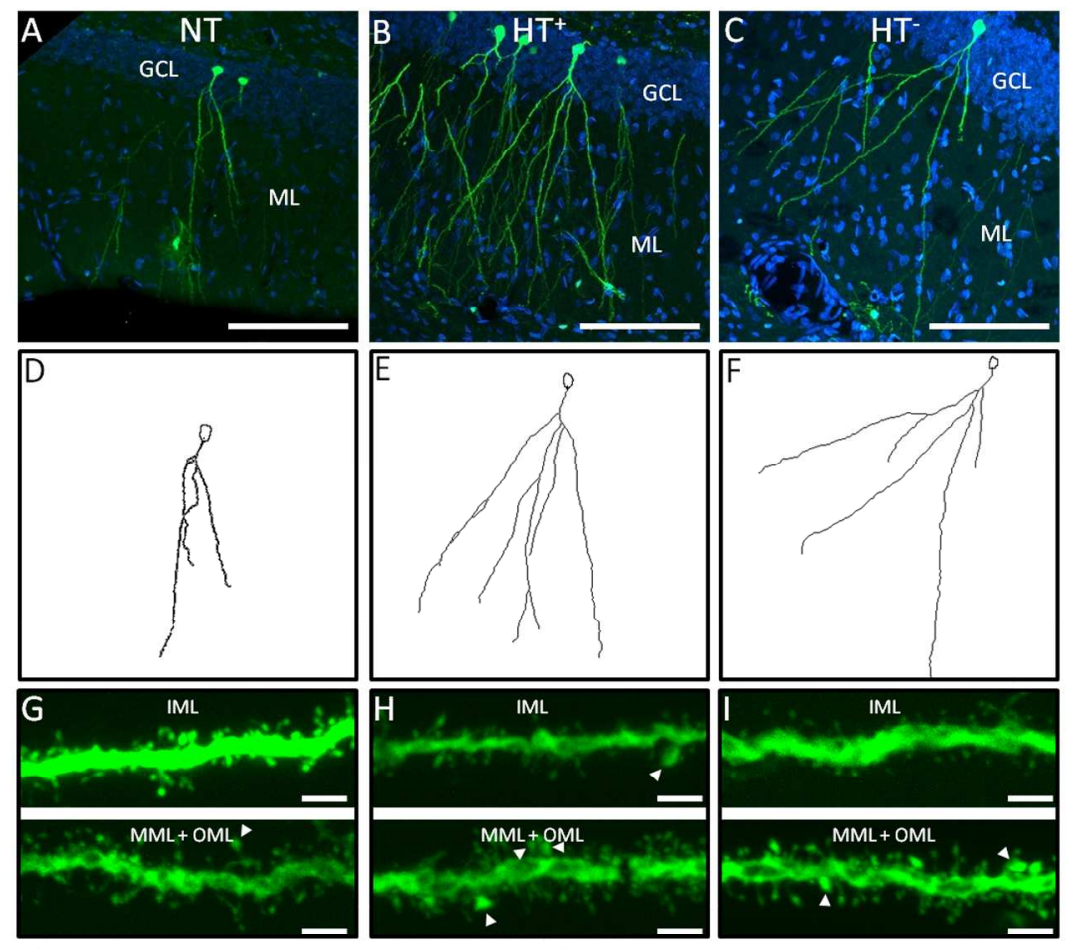

J
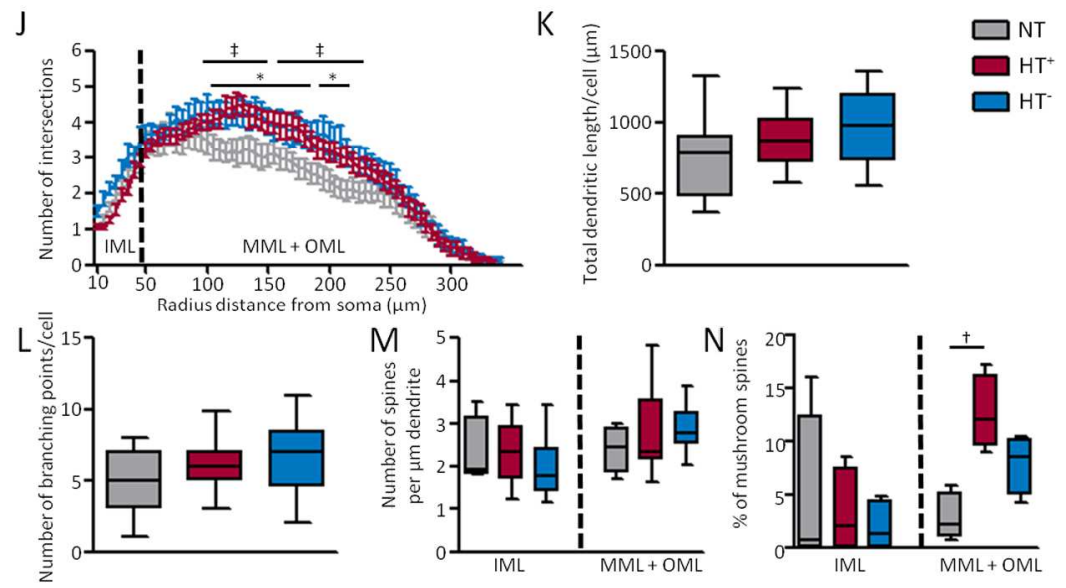

Figure 4: At eight weeks of development, post-seizure born DGC show increased dendritic complexity and altered spine morphology. Photomicrographs of hippocampal DGC, born and eGFP labeled $24 \mathrm{~h}$ after $\mathrm{NT}(\mathrm{A}), \mathrm{HT}^{+}$(B) or $\mathrm{HT}^{-}$(C) treatment and visualized at P66. Examples of dendritic traces $(D-F)$ that were acquired from respective photomicrographs $(A-C)$. Representative pictures of dendritic spines in the IML and MML+OML after NT $(\mathrm{G}), \mathrm{HT}^{+}(\mathrm{H})$ or $\mathrm{HT}^{-}(\mathrm{I})$ treatment. Arrowheads indicate mushroom type spines. Sholl analysis of dendritic complexity of eGFP labelled DGC (J). Quantification of total dendritic length (K) and number of branching points (L) of eGFP labelled DGC. Quantitative analysis of the overall spine density in dendritic segments located in the IML and the MML+OML (M). Quantitative analysis of the mushroom type spine density in dendritic segments located in the IML and the MML+OML (N). Data are represented as median (black bar), 25-75 percentile (box) and 5-95 percentile (whiskers; K, L, M, N) or as mean \pm SEM (J). * ${ }^{*} \mathrm{HT}^{+}$vs NT, $\mathrm{p}<0.01 ; \neq=\mathrm{HT}^{+}$vs $\mathrm{HT}^{-}, \mathrm{p}<0.01 ;+=\mathrm{HT}^{+}$vs NT, $\mathrm{p}<0.05 ; \mathrm{HT}^{+} \mathrm{n}=4, \mathrm{HT}^{-}$ $\mathrm{n}=4, \mathrm{NT} n=4$. $\mathrm{GCL}=$ granular cell layer, $\mathrm{IML}=$ inner molecular layer, $M M L=$ middle molecular layer, $\mathrm{OML}=$ outer molecular layer. Scale bars: A-C $50 \mu \mathrm{m}, \mathrm{G}-\mathrm{I} 5 \mu \mathrm{m}$. 


\section{Page 27 of 25}

Epilepsia

1

2

3

4

$267 \times 383 \mathrm{~mm}(300 \times 300$ DPI $)$

5

6

7

10

11

12

13

14

15

16

17

18

19

20

21

22

23

24

25

26

27

28

29

30

34

35

36

37

38

39

40

41

42

43

44

45

46

47

48

49

50

51

52

53

54

55

56

57

58

59

60 